\title{
London force and energy transportation between interfacial surfaces
}

\author{
David S. Bradshaw, Jamie M. Leeder, Justo Rodríguez and David L. Andrews \\ Nanostructures and Photomolecular Systems, School of Chemical Sciences, \\ University of East Anglia, Norwich NR4 7TJ, United Kingdom
}

\begin{abstract}
With appropriately selected optical frequencies, pulses of radiation propagating through a system of chemically distinct and organized components can produce areas of spatially selective excitation. This paper focuses on a system in which there are two absorptive components, each one represented by surface adsorbates arrayed on a pair of juxtaposed interfaces. The adsorbates are chosen to be chemically distinct from the material of the underlying surface. On promotion of any adsorbate molecule to an electronic excited state, its local electronic environment is duly modified, and its London interaction with nearest neighbor molecules becomes accommodated to the new potential energy landscape. If the absorbed energy then transfers to a neighboring adsorbate of another species, so that the latter acquires the excitation, the local electronic environment changes and compensating motion can be expected to occur. Physically, this is achieved through a mechanism of photon absorption and emission by molecular pairs, and by the engagement of resonance transfer of energy between them. This paper presents a detailed analysis of the possibility of optically effecting such modifications to the London force between neutral adsorbates, based on quantum electrodynamics (QED). Thus, a precise link is established between the transfer of excitation and ensuing mechanical effects.
\end{abstract}

Keywords: London forces, quantum electrodynamics, resonance energy transfer, adsorbates, interfaces, NEMS.

\section{INTRODUCTION}

Resonance energy transfer (RET), the transportation of electronic excitation between ions, atoms, or molecules following photoexcitation, is a mechanism of remarkably wide relevance across a wide range of physical, chemical and biological systems - see for example refs [1-4]. However modifications to the London dispersion interaction between energy donor and acceptor units necessarily accompany the operation of RET. Until now, it appears that little regard has been given to the resulting mechanical effects.

The dispersion interaction is most accurately described in terms of the Casimir-Polder potential ${ }^{5}$; using quantum electrodynamics (QED), its explicit form emerges from calculations based on intermolecular coupling through virtual photon mediators ${ }^{6}$. Although the long-range behavior of the leading contribution to the potential runs with the inverse seventh power of the inter-particle distance $\mathrm{R}$, the shorter-range form that operates over distances where effects are most pronounced exhibits an $\mathrm{R}^{-6}$ asymptotic behavior. The latter attractive form of interaction, due to induced dipole-induced dipole coupling, is usually known as the London potential. Although this potential is usually considered as an interaction between molecules in their ground states, a potential of similar form may readily be derived for molecules in excited states ${ }^{7}$.

Since the form of the dispersion interaction depends on the electronic states of the molecular participants, the dispersion force between neutral molecules is clearly subject to change during the course of RET. The associated modification of electromagnetic interactions between the donor and other units will generally produce modified intermolecular forces, resulting in a degree of local movement as the system becomes accommodated to the new potential energy field. In particular, in an environment where intermolecular forces are balanced in a stable equilibrium configuration, any changes associated with the migration of local electronic excitation should effectively act as a small perturbation to the equilibrium of intrinsic forces, producing measurable displacements.

This paper reports the results of preliminary calculations casting a new light on the physical link between these features. First, a succinct theoretical treatment of the dispersion pair potential is given, in which the interaction energy dependence on the electronic state of the interacting particles is explicitly delivered. Since any adaptation to subtle features in the changing force fields should be most readily testable in an ensemble, rather than in individual particle pairs, the results are then applied to an experimentally meaningful system, in which the two units between which energy 
is transferred are surface adsorbates. The latter are assumed to be arrayed on a pair of juxtaposed interfaces in close proximity. The theory is further developed to elicit the dynamical behavior. Here the developing response of the system, following initial excitation, is ascertained as a function of time. Possible developments of the theory are then discussed in the Conclusion.

\section{METHODOLOGY}

The dispersion potential is characterized by interactions between molecules in the short-range region, i.e. at distances beyond significant orbital overlap. In the formal theory of QED, these interactions are considered to derive from an exchange of two virtual photons between the participant molecules. A variety of methods is available for the detailed calculations $^{7-9}$ but in each case, in order to secure results applicable to arbitrarily large distances, considerable complexity is entailed. For present purposes, however - where the focus is on interactions within the Förster radius, and therefore over distances where retardation effects are negligible - a simpler and much more direct method can be employed, as shown below.

\subsection{Pairwise dispersion interaction}

To introduce the methods to be employed below, consider first a simple pairwise coupling between a donor $A$ and acceptor $B$, both having permanent electric dipoles. In a QED derivation of the coupling, the interaction is represented as a virtual photon transfer between $A$ and $B$, accommodating retardation effects. However, within the near-field range of interest for the present calculations, the virtual photon creation and annihilation events may be treated as essentially simultaneous, and the calculation can be treated by first-order perturbation theory. The result is secured using a pairwise operator, $\mathbf{W}_{A B}$, given explicitly by;

$$
\mathbf{W}_{A B}=\frac{\mu_{i}^{A} \mu_{j}^{B}}{4 \pi \varepsilon_{0} R^{3}}\left(\delta_{i j}-3 \hat{R}_{i} \hat{R}_{j}\right)
$$

Here, $\boldsymbol{\mu}^{\xi}$ is a dipole moment operator which operates on the states of molecule $\xi$, and $\mathbf{R}$ is the intermolecular vector; summation over repeated Cartesian indices is implied. The pair interaction potential is thus determined by $\Delta E=\left\langle\Lambda\left|\mathbf{W}_{A B}\right| \Lambda\right\rangle$. In the latter, $|\Lambda\rangle$ signifies the unperturbed basis state involving the donor molecule in state $a$, the acceptor in state $b$ (plus the vacuum radiation field, i.e. no input radiation present), the product state representable as $|\Lambda\rangle=\left|A^{a} ; B^{b}\right\rangle$. Therefore, the interaction potential emerges from (1) with the diagonal matrix elements $\mu_{i}^{a a(A)} \equiv\left\langle A^{a}\left|\mu_{i}^{A}\right| A^{a}\right\rangle$ and $\mu_{j}^{b b(B)}$ (i.e. the static dipole moments) substituting for the dipole operators.

The dispersion interaction is an additional form of coupling which, in the case of interaction between non-polar molecules, becomes the dominant form - this is the focus of the studies reported below. The coupling derives from second-order perturbation theory, i.e.;

$$
\Delta E=\sum_{S} \frac{\left\langle\Lambda\left|\mathbf{W}_{A B}\right| S\right\rangle\left\langle S\left|\mathbf{W}_{A B}\right| \Lambda\right\rangle}{E_{\Lambda}-E_{S}}
$$

where $|S\rangle$ is an intermediate or virtual state of the system. On substitution of equation (1) into (2), with the state of each component duly specified, the following emerges; 


$$
\begin{aligned}
\Delta E\left(A^{a} B^{b}\right) & =\sum_{r, s} \frac{\left\langle A^{a} ; B^{b}\left|\mathbf{W}_{A B}\right| A^{r} ; B^{s}\right\rangle\left\langle A^{r} ; B^{s}\left|\mathbf{W}_{A B}\right| A^{a} ; B^{b}\right\rangle}{E_{a}^{A}+E_{b}^{B}-E_{r}^{A}-E_{s}^{B}} \\
& =\frac{1}{16 \pi^{2} \varepsilon_{0}^{2} R^{6}}\left[\frac{\mu_{i}^{a r(A)} \mu_{j}^{b s(B)} \mu_{k}^{r a(A)} \mu_{l}^{s b(B)}\left(\delta_{i j}-3 \hat{R}_{i} \hat{R}_{j}\right)\left(\delta_{k l}-3 \hat{R}_{k} \hat{R}_{l}\right)}{E_{a r}^{A}+E_{b s}^{B}}\right],
\end{aligned}
$$

with summation effected over molecular states $\left|A^{r}\right\rangle$ and $\left|B^{s}\right\rangle$. Whilst (2) is generally valid for rigidly oriented molecules, the key features of the physics are made more transparent through the simplification which results from performing an orientational average. As is assumed in the following model system calculations, the dipole moments of both the donor and acceptor are thus considered to be randomly oriented, in situ. With the isotropic average applied to the result emerging from equation (3) the following is ascertained;

$$
\Delta E\left(A^{a} B^{b}\right)=-\frac{1}{24 \pi^{2} \varepsilon_{0}^{2} R^{6}} \sum_{r, s} \frac{\left|\boldsymbol{\mu}_{A}^{a r}\right|^{2}\left|\boldsymbol{\mu}_{B}^{b s}\right|^{2}}{E_{r a}^{A}+E_{s b}^{B}},
$$

which reduces to the well-known London formula when $a$ and $b$ are ground levels. In the latter case each $E_{r a}^{A}$ and $E_{s b}^{B}$ is positive and, therefore, the result of equation (4) is invariably a negative quantity. With due regard to the inverse power dependence on distance, the attractive nature of the dispersion potential is thus apparent. In the following, as an expedient but very good approximation, we shall assume that the state summations in equation (4) are limited to the three states that determine the most prominent optical features. Thus, the donor and acceptor are chosen to have the significant molecular state labels $r \in\left\{0, \alpha, \alpha^{*}\right\}$ and $s \in\left\{0, \beta, \beta^{*}\right\}$. Of these, the states $\left|A^{\alpha^{*}}\right\rangle$ and $\left|B^{\beta^{*}}\right\rangle$ are specifically included as representatives of unpopulated molecular states.

\subsection{Ensemble dispersion interaction}

Moving beyond a single pair interaction, the next step is to develop the kinetics for an ensemble of donors and acceptors with $N$ molecules of each, counter-positioned across a gap set up in a vacuum or in air. To determine the average dispersion potential, $\overline{\Delta E}$, for this system, a sum of $\Delta E\left(A^{a} B^{b}\right)$ over all possible state combinations is required. Overall there are four permutations to be taken into account, and from (4) the ensemble energy emerges as follows;

$$
\overline{\Delta E}=-\frac{1}{24 \pi^{2} \varepsilon_{0}^{2} R^{6}} \sum_{\substack{a=0, \alpha, b=0, \beta}} N_{a}^{\prime} N_{b}^{\prime \prime} \sum_{\substack{r=0, \alpha, \alpha^{*}, s=0, \beta, \beta^{*}}} \frac{\left|\boldsymbol{\mu}_{A}^{a r}\right|^{2}\left|\boldsymbol{\mu}_{B}^{b s}\right|^{2}}{E_{r a}^{A}+E_{s b}^{B}} .
$$

In the first summation on the right-hand side of equation (5), $N_{a}^{\prime}$ and $N_{b}^{\prime \prime}$ are the fractional populations of $A$ in state $a$ and $B$ in state $b$, respectively; the time-dependence of $\overline{\Delta E}$ will emerge from the temporal behavior of these populations, modeled later in Section 2.4. By the rules of perturbation theory, the terms in which $a=r$ and $b=s$ are excluded in the second summations of equation (5). 


\subsection{Interface dispersion interaction}

The last stage, in the development of results for a meaningfully scaled system, is to extend consideration to a structurally ordered implementation of the previous ensemble. This involves two parallel square-based arrays - one comprising donors and the other, acceptors - displaced by a distance $d$ and each composed of $N$ molecules (Fig. 1).

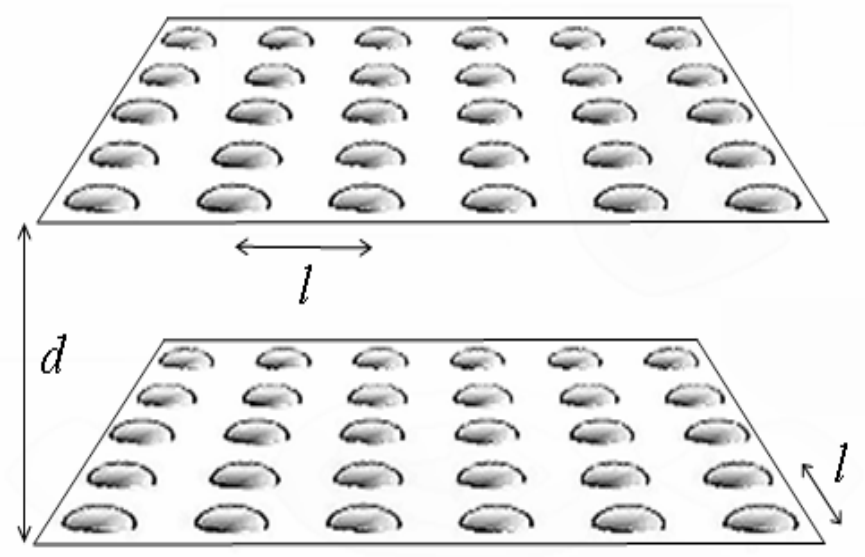

Fig. 1. Pair of parallel arrays displaced from each other by $d$. Each array is composed of identical molecules on a square lattice, a distance $l$ apart.

Within each plane, these molecules are assumed to be chemically identical and equally spaced from their nearest neighbors by a lattice constant, $l$; a chemical difference in the donors and acceptors achieves a spectroscopic gradient that precludes back-transfer. Determining the dispersion potential per donor, $\overline{\Delta E}$, for the array system, the following is established;

$$
\begin{aligned}
\overline{\Delta E} & =-\frac{1}{24 \pi^{2} \varepsilon_{0}{ }^{2} l^{6}} \sum_{\substack{a=0, \alpha, b=0, \beta}} N_{a}^{\prime} N_{b}^{\prime \prime} \sum_{\substack{r=0, \alpha, \alpha^{*}, s=0, \beta, \beta^{*}}} \frac{\left|\boldsymbol{\mu}_{A}^{a r}\right|^{2}\left|\boldsymbol{\mu}_{B}^{b s}\right|^{2}}{E_{r a}^{A}+E_{s b}^{B}} \int_{-\infty}^{\infty} \int_{-\infty}^{\infty} \frac{1}{\left(d^{\prime 2}+n^{2}+m^{2}\right)^{3}} \mathrm{~d} n \mathrm{~d} m \\
& =-\frac{1}{48 \pi \varepsilon_{0}{ }^{2} l^{2} d^{4}} \sum_{\substack{a=0, \alpha, b=0, \beta}} N_{a}^{\prime} N_{b}^{\prime \prime} \sum_{\substack{r=0, \alpha, \alpha^{*}, s=0, \beta, \beta^{*}}} \frac{\left|\boldsymbol{\mu}_{A}^{a r}\right|^{2}\left|\boldsymbol{\mu}_{B}^{b s}\right|^{2}}{E_{r a}^{A}+E_{s b}^{B}},
\end{aligned}
$$

where $R^{2}=d^{2}+(n l)^{2}+(m l)^{2}$, with $n$ and $m$ integers. In addition, $d^{\prime}=d / l$ denotes the aspect ratio of the planar array, and the double integration over $n$ and $m$, treated as continuous variables, is a suitable approximation for summation over all molecules in the acceptor array - exhibiting the fact that equation (6) describes the dispersion energy associated with each single donor in its interaction with the entire acceptor array (of potentially infinite size although, in view of the distance dependence, generally dominated by a few close neighbors); it is not simply a sum over specifically correlated $A-B$ pairs.

\subsection{Modelling dynamic behavior}

To numerically evaluate changes in the array dispersion as defined by throughout RET, the time-evolving populations $N_{a}^{\prime}$ and $N_{b}^{\prime \prime}$ need to be determined. Let us now consider a simple sequence of photophysical interactions engaging an interplay of the three-state molecules introduced in the last section. We assume that resonant laser light produces an 
initial excitation in $A$, between the ground state and vibrational levels of an electronic excited state $\alpha$. The optical input is such that the incident photons exceed the energy for $B$ to undergo transition to the first excited state $\beta$, but that their energy is insufficient to excite the higher states $\alpha^{*}$ or $\beta^{*}$; the latter are not populated under these conditions. Beyond initial excitation, the input plays no further part in any subsequent processes.

The mechanism that follows is summarised in Fig. 2. The first step is internal vibrational relaxation (IVR), dissipating part of the energy acquired by $A$. The molecule can subsequently relax to the electronic ground state through a variety of mechanisms - spontaneous emission, RET, internal conversion, etc. For simplicity, all such electronic relaxation processes, with exception of RET, are included in a representative rate constant $k_{r e l}^{\alpha}$. RET between $A$ and $B$ generally signifies that a downward transition occurs in $A$, to a vibrational level of the ground electronic state, while the released energy produces an upward transition in molecule $B$, from its ground state to a vibrational level of the electronic state $\beta$. In the quantum amplitude for RET, energy conservation is ensured by the frequency-weighted overlap between the absorption spectrum of the acceptor and the emission spectrum of the donor, accommodating all possible routes for the energy relocation. Generally, the 'forward' process is efficiently achieved when there is a spectroscopic gradient in the direction of the transfer, $E_{\alpha 0}>E_{\beta 0}$, reducing significantly 'backward' RET ${ }^{10}$; the low probability of the latter is signified by its neglect in the figure and in subsequent calculations. The last stage of the process is the vibrational and electronic relaxation of $B$. Here, all relaxation processes are accommodated by a representative rate constant $k_{r e l}^{\beta}$.

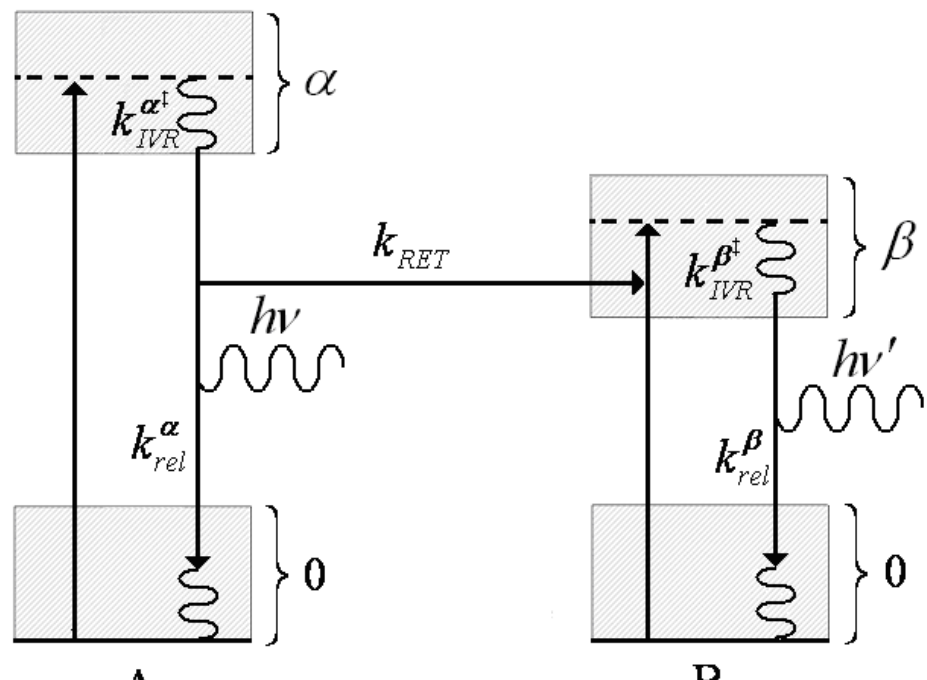

A

$\mathrm{B}$

Fig. 2. Jablonski diagram, the rate constants $k$ denoting processes determining the excited populations of molecules $A$ and $B$. These processes involve amongst others, spontaneous emission of photons, $h v\left(k_{r e l}^{\alpha}\right)$ from $A$ and lower energy emission (owing to IVR) $h v^{\prime}\left(k_{r e l}^{\beta}\right)$ from $B$.

It is worth observing a simplifying feature that arises when more than one donor (or acceptor) is present. Consider, for example, the implications of having several chemically equivalent donors, in close proximity to a given donor $A$. In principle, RET among these molecules is also possible. However, the rapid IVR that follows initial donor excitation will generally put that molecule into an energy level where its decay has relatively small overlap with the red end of a neighbor's absorption profile. In the competing process of RET to a nearby acceptor, the associated spectroscopic gradient will generally engender a much larger spectral overlap and hence a significantly larger transfer rate.

The time-resolved change in the population of the initial excited state can be determined by analysis of all decay routes of $\alpha$; as shown in Fig. 2 we have: 


$$
\frac{\mathrm{d}}{\mathrm{d} t} N_{\alpha}^{\prime}=-\left(k_{r e l}^{\alpha}+k_{R E T}\right) N_{\alpha}^{\prime}
$$

The above differential equation is solved under the condition that the population of state $\alpha$ is assigned an initial arbitrary value:

$$
N_{\alpha}^{\prime}(t)=N_{\alpha}^{\prime}(0) \exp ^{-t\left(k_{r e l}^{\alpha}+k_{R E T}\right) N_{\alpha}^{\prime}}
$$

By application of the physically reasonable conditions that $k_{I V R}^{\beta^{\sharp}}>k_{R E T}, N_{\alpha}^{\prime}>N_{\beta^{\ddagger}}^{\prime \prime}$ and $\beta(0)=0$, the ensuing growth in population of $\beta$ is seen to be dominated by $k_{R E T}$. The following expression represents the time-dependent variation in $N_{\beta}^{\prime \prime}$ :

$$
\frac{\mathrm{d}}{\mathrm{d} t} N_{\beta}^{\prime \prime}=k_{R E T} N_{\alpha}^{\prime}-k_{r e l}^{\beta} N_{\beta}^{\prime \prime}
$$

\section{RESULTS}

The modeled fractional populations for $\alpha$ and $\beta$ are shown in Fig. 3, with the initial value $N_{\alpha}^{\prime}(0)$ set to 1 . It should be emphasized that a result normalized in this sense is not meant to signify the experimentally unrealistic excitation of all donor molecules within the system. Rather, it is a device serving as a reminder that the calculations simply portray the relationship between donor decay and acceptor excitation through RET. The excited state donor lifetime is modeled as $1 \mathrm{~ns}$ and $k_{R E T}$ accounts for the majority of the total decay of $\alpha$, relative to $k_{r e l}^{\alpha}$. Such a condition represents a sufficiently strong short-range interaction between $A$ and $B$, that RET is the dominant decay process for $\alpha$ - as is the case for donoracceptor pairs within the Förster radius.

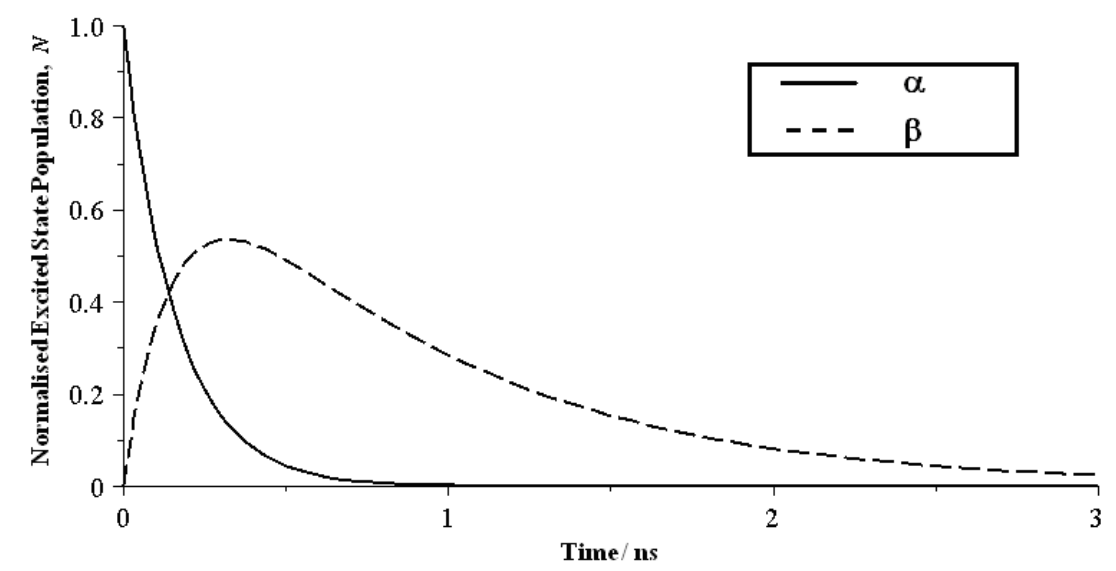

Fig. 3. Representative $N_{\alpha}^{\prime}$ and $N_{\beta}^{\prime \prime}$ excited state population profiles following initial pulsed laser excitation. Values of $k_{R E T}$ and $k_{r e l}^{\alpha}$ are chosen such that $k_{R E T}$ accounts for $80 \%$ of the total decay of $N_{\alpha}^{\prime} ; k_{r e l}^{\alpha}$ and $k_{r e l}^{\beta}$ are taken as equal. 
From equation (6), numerical results can now be calculated to evaluate the physical consequences of electronic energy flow into, across, and out of the two arrays, see Fig. 4. Key determinants of the outcome are the magnitudes of the transition dipole moments $\boldsymbol{\mu}_{A}^{a r}$ and $\boldsymbol{\mu}_{B}^{b s}$, here for convenience set equal and ranging over modest values between 3 and $5 \mathrm{D}$. A figure of $1.0 \mathrm{~nm}$ is adopted for both $l$ and $d$. Transition energies to $\left|A^{\alpha^{*}}\right\rangle$ and $\left|A^{\alpha}\right\rangle$, from $\left|A^{0}\right\rangle$ are selected to correspond with wavelengths of $300 \mathrm{~nm}$ and $350 \mathrm{~nm}$ respectively. Transitions from $\left|B^{0}\right\rangle$ to $\left|B^{\beta^{*}}\right\rangle$ and $\left|B^{\beta}\right\rangle$ are lower in energy, wavelengths of $400 \mathrm{~nm}$ and $450 \mathrm{~nm}$ accordingly.

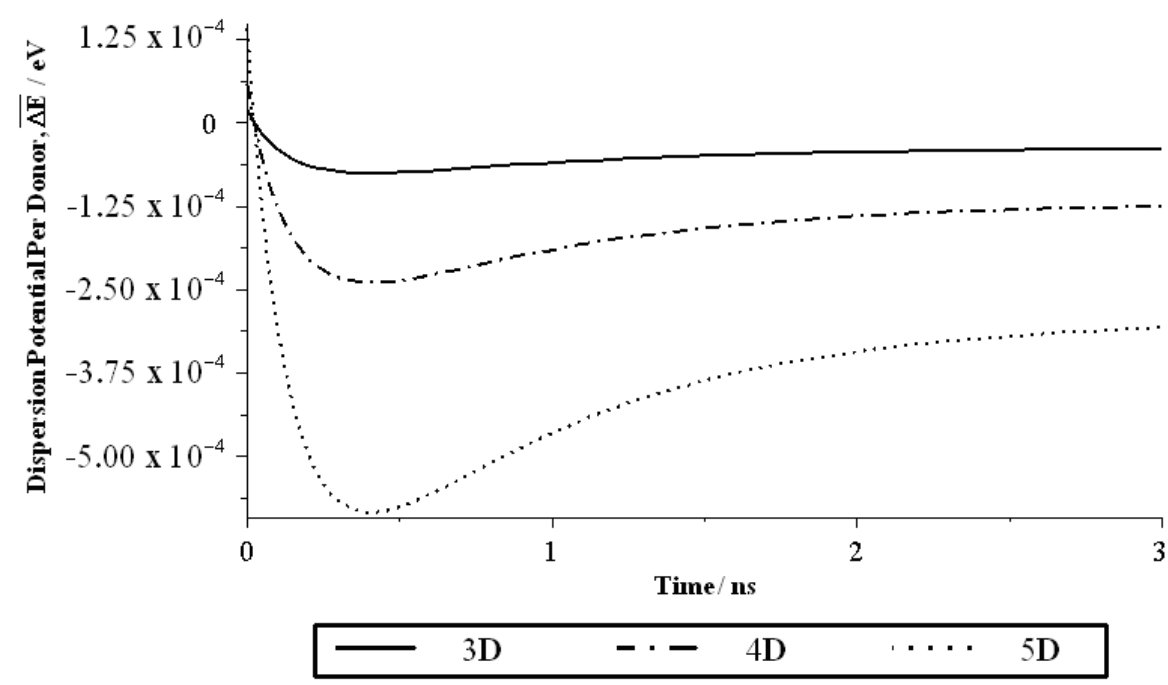

Fig. 4. Evolution of the optically modified donor-acceptor interaction as a function of time, following pulsed laser excitation.

After donor electronic excitation, a decrease in population of $\left|A^{\alpha}\right\rangle$ results in a corresponding drop in the potential energy of interaction between donor and acceptor arrays. Analyzing the results from Figs 3 and 4 , it can be observed that the interaction energy reaches a minimum as the population of $\left|B^{\beta}\right\rangle$ approaches a maximum. The timedependence of each excited state is tempered by the various losses that lead to eventual decay. Over this interval, compensating motions return the system to its ground-state interaction potential. To quantify the corresponding ensemble-averaged forces involved, per donor, we develop equation (6) into $\mathbf{F}_{a v e}=-(\partial \overline{\Delta E} / \partial d) \hat{\mathbf{n}}$, where $\hat{\mathbf{n}}$ is the normal to the plane of each array. For the $\overline{\Delta E}$ values presented in Fig. 4, the dispersion force for the donor-acceptor ensemble model varies in the picoNewton range. These figures are highly encouraging; with the rapidly ongoing development of techniques such as atomic force spectroscopy (AFM) ${ }^{11,12}$, such forces easily fall into the range of possible measurement.

\section{CONCLUSIONS}

In the study of interfaces and surface adsorbates, molecules generally reside in their electronic ground state and it is not surprising to find that the familiar forms of intermolecular potential are commonly adopted with that implicit assumption. Under conditions of thermal equilibrium, electronically excited state populations are usually vanishingly small, while even under the conditions that apply in active surface experimentation, the excited populations are, on the whole, severely limited in spatial and temporal extent. Nonetheless, the nature of interaction between neutral molecules certainly varies, even within those small regions of time and space, according to the electronic state.

This paper has sought to address, and to begin to quantify, the key issues that surround modifications to the London dispersion force, arising through the operation of RET. The first aim has been to analyze those changes by 
consideration of pair intermolecular forces, using a robust QED foundation. Secondly, by application to a simple model system, the analysis has aimed to illustrate the practicality of measuring the shifts in energy and force that must accompany energy transfer in any multi-component system. In a system that displays typical RET behavior, specific calculations based on an array configuration have exhibited a characteristic mechanical response and recovery. The effects are striking, and should prove amenable to measurement by currently available instrumentation.

Some issues invite future work to prompt and more accurately simulate experimentation. For example, at the price of considerable additional complexity, a more precise theoretical model might disengage the simplifying assumptions of ensemble average orientations, or the notionally perfect planarity of the donor and acceptor arrays. In comparison to the present predictions, based on isotropic averaging, the inclusion of orientational order in the calculations is expected to be reflected in an increase in the mechanical response. Hence, it is likely that the response will be even more readily detectable than the conservative estimates reported here.

Work currently underway is further developing this model to incorporate additional optical phenomena. One example is the effect of intense off-resonant laser light in promoting increased RET efficiency, by what is termed laserassisted resonance energy transfer (LARET) ${ }^{13}$. Any resulting enhancements to the rate of RET should have a noticeable effect in producing additional modifications to the intermolecular dispersion forces. It is our hope and intention that work on these issues will play a role in developing and linking the theory into practical applications.

\section{ACKNOWLEDGEMENTS}

The authors are grateful to the EPSRC and the Leverhulme Trust for financing this work.

\section{REFERENCES}

[1] Sapsford, K. E., Berti, L. and Medintz, I. L., "Materials for fluorescence resonance energy transfer analysis: Beyond traditional donor-acceptor combinations," Angew. Chem., 45, 4562-4589 (2006).

[2] Andrews, D. L., Li, S., Rodríguez, J. and Slota, J., "Development of the energy flow in light-harvesting dendrimers," J. Chem. Phys., 127, 134902 (2007).

[3] Polivka, T., Hiller, R. G. and Frank, H. A., "Spectroscopy of the peridinin-chlorophyll-a protein: Insight into lightharvesting strategy of marine algae," Arch. Biochem. Biophys., 458, 111-120 (2007).

[4] Farinha, J. P. S. and Martinho, J. M. G., [Fluorescence of supermolecules, polymers, and nanosystems], Springer, Berlin, 215-255 (2008).

[5] Casimir, H. B. G. and Polder ,D., "The influence of retardation on London-van der Waals forces,” Phys. Rev., 73. 360-372 (1948).

[6] Power, E. A. and Thirunamachandran, T., "Casimir-Polder potential as an interaction between induced dipoles," Phys. Rev. A, 48, 4761 (1993).

[7] Power, E. A. and Thirunamachandran, T., "Dispersion forces between molecules with one or both molecules excited," Phys. Rev. A, 51, 3660 (1995).

[8] Salam, A., "A general formula obtained from induced moments for the retarded van der Waals dispersion energy shift between two molecules with arbitrary electric multipole polarizabilities: I. Ground state interactions," J. Phys. B: At. Mol. Opt. Phys., 39, S651-S662 (2006).

[9] Salam, A., "A general formula obtained from induced moments for the retarded van der Waals dispersion energy shift between two molecules with arbitrary electric multipole polarizabilities: II. Excited state interactions," J. Phys. B: At. Mol. Opt. Phys., 39, S663-S670 (2006)

[10] Andrews, D. L. and Rodríguez, J., "Resonance energy transfer: Spectral overlap, efficiency, and direction,” J. Chem. Phys., 127, 084509 (2007).

[11] Baumgartner, W., Hinterdorfer, P. and Schindler, H., "Data analysis of interaction forces measured with the atomic force microscope," Ultramicroscopy, 82, 85-95 (2000).

[12] Hugel, T. and Seitz, M., "The study of molecular interactions by AFM force spectroscopy," Rapid Commun., 22, 989-1016, (2001).

[13] Allcock, P., Jenkins, R. D. and Andrews, D. L., "Laser-assisted resonance-energy transfer," Phys. Rev. A, 61, 023812 (2000). 